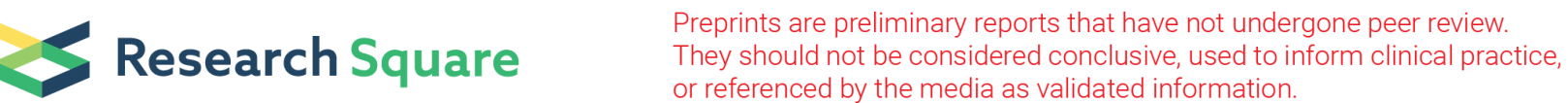

\section{Virus discharge and initial gastrointestinal involvement are negatively associated with circulating lymphocyte count in COVID-19}

\section{Wei Chen}

The second affiliated hospital of wenzhou medical university

Kenneth I. Zheng

The First affiliated hospital of wenzhou medical university

Saiduo Liu

The sixth people's hospital of wenzhou

Chongyong Xu

The second affiliated hospital of wenzhou medical university

Chao Xing

The second affiliated hospital of wenzhou medical university

Zengpei Qiao ( $\square$ Zengpei_qiao@wzhealth.com )

The second affiliated hospital of wenzhou medical university https://orcid.org/0000-0002-6668-4404

\section{Research Article}

Keywords: circulating lymphocyte count, COVID-19, gastrointestinal tract, CRP, stool

Posted Date: July 7th, 2020

DOI: https://doi.org/10.21203/rs.3.rs-40462/v1

License: () (1) This work is licensed under a Creative Commons Attribution 4.0 International License.

Read Full License 


\section{Abstract}

Background: It's reported SARS-CoV-2 could transmit via gastrointestinal tract, with or without pulmonary symptoms. However, as far as we know, there is no convenient marker to predict the virus discharge in stool and initial gastrointestinal involvement of COVID-19.

Aims: We aimed to investigate the biomarker predicting virus discharge in stool and initial gastrointestinal involvement of COVID-19, which may assist the clinicians to better combat and prevent COVID-19.

Methods: The patients complained of initial gastrointestinal involvement, including vomiting, diarrhea, with or without respiratory symptoms, attending the Sixth People's Hospital of Wenzhou, and the Second Affiliated Hospital of Wenzhou Medical University, were screened by qRT-PCR for SARS-CoV-2. The Confirmed COVID-19 patients without contaminated ingestion were all enrolled to investigate the association between circulating lymphocyte count and virus discharge, initial gastrointestinal involvement.

Results: 76 COVID-19 patients were finally enrolled in this study (mean age 44.5, male 44.7\%), with 24 $(31.5 \%)$ complained of initial gastrointestinal symptoms. Significantly lower circulating lymphocyte count was found in the patients with positive results of qRT-PCR on stool $(p=0.012)$. Furthermore, when the circulating lymphocyte count increased from bottom tertile to the $2^{\text {nd }}$ and $3^{\text {rd }}$ tertiles, the risk of initial gastrointestinal involvement decreased by nearly $75 \%(\mathrm{OR}=0.25,95 \% \mathrm{Cl}: 0.07,0.98, \mathrm{p}=0.047), 83 \%(\mathrm{OR}$ $=0.17,95 \% \mathrm{Cl}: 0.05,0.63, \mathrm{p}=0.008)$, adjusted for possible confounders.

Conclusions: The circulating lymphocyte count is negatively associated with virus discharge in stool in COVID-19 patients, and the risk of initial gastrointestinal involvement also.

\section{Introduction}

The Coronavirus (CoVs) belongs to the subfamily of Ortho-coronavirinae in the family of Coronaviridae and the Order Nidovirales. In 2003, a SARS-CoV had caused the outbreak of severe acute respiratory syndrome (1). The virus responsible for the epidemic worldwide in late 2019, has been identified as novel coronavirus by World Health Organization (WHO), and termed as SARS-CoV-2, characterized as highly contagious and deadly. By the end of June 2020, more than 10 million cases infected with SARS-CoV-2, and more than 500 thousand of deaths were confirmed worldwide, and the COVID-19 has been declared a pandemic by WHO.

Since it is one of the respiratory pathogens, COVID-19 is mainly transmitted through respiratory droplet. Therefore, great efforts have been focused on the respiratory manifestations and transmission of COVID-19, and it made some differences to the recovery and prevention of COVID-19. However, the COVID-19 patients probably continue to discharge virus via GI tract even after pulmonary symptoms have resolved (2). What is worse, SARS-CoV-2 has shown the probability to initially affect 
gastrointestinal $(\mathrm{Gl})$ tract. There are even no symptoms of respiratory system in some patients with positive signals of SARS-CoV-2 in stool $(3,4)$. This indicates probable virus discharge in the lack of respiratory symptoms, which makes it difficult to diagnose and combat CVOID-19.

Studies have shown that inflammatory cells effusion and lower lymphocyte is associated with severe respiratory syndrome in the process of COVID-19. We therefore hypothesized that lower circulating lymphocyte (CLC) might predict the initial GI involvement and virus discharge in stool. Our results would assist clinicians to better combat and prevent COVID-19 dissemination.

\section{Materials And Methods}

\section{Selection and Description of Participants}

Anonymous clinical and laboratory data were retrospectively collected and analyzed to facilitate better prediction of initial GI involvement, COVID-19 treatment and virus transmitting prevention. The patients complained of initial GI involvement, including vomiting, diarrhea, and respiratory symptoms including cough, chest pain and other extra-pulmonary symptoms, with or without fever, attending the Sixth People's Hospital of Wenzhou, and the Second Affiliated Hospital of Wenzhou Medical University, were screened by qRT-PCR on swab for SARS-CoV-2. Diagnostic criteria for COVID-19 is based on the CDCP (China) Diagnosis and Treatment of COVID-19 (5). All the patients of positive results were enrolled. The exclusion criterium is contaminated ingestion ahead of admission. And finally, all data except the result of nucleic acid in stool were collected at baseline. The results of qRT-PCR on stool were collected in serial until it changed into negative.

\section{Blood Routine Test and Serologic Test}

Fasting venous blood samples were collected and analyzed by standard method in the clinical laboratory center. Blood routine test was performed to count the blood cells and white blood cell classification, to measure C-reactive protein and hemoglobin concentration in the Mindray BC-5390 system (Shenzhen, China). The biochemical tests were performed in the VITROS 5600 Integrated Immunodiagnostic System (VITROS 5600, Johnson, New Jersey, U.S. A.), including albumin, total protein, lactic dehydrogenase, creatine kinase, alanine aminotransferase, aspartate transaminase, total bilirubin.

\section{Inspection of Virus Discharge in Stool}

A ReadyPrep Prot RNA Extract Kit (Sol/Insol, BioRad) was used to extract total RNA from $200 \mu$ of stool supernatant, following the manufacturers' instructions as mentioned elsewhere. RNA was eluted in 50 $\mu$ l of elution buffer and immediately used as the template for RT-PCR detecting SARS-CoV-2, which could be found in previous studies. Briefly, RNA template was added in the qPCR system using HiScript II One

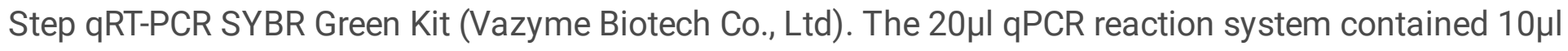




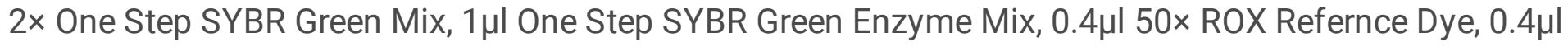
of each primer $(10 \mu \mathrm{M})$ and $2 \mu \mathrm{l}$ RNA. The following program was performed for amplification: $50{ }^{\circ} \mathrm{C}$ for 3 min, $95^{\circ} \mathrm{C}$ for $30 \mathrm{~s}$ followed by 40 cycles consisted of $95^{\circ} \mathrm{C}$ for $10 \mathrm{~s}, 60^{\circ} \mathrm{C}$ for $30 \mathrm{~s}$, and a default melting curve step in an ABI 7500 machine (6).

\section{Statistics}

Statistical data analysis was performed by using SPSS 25.0 (IBM, New York, U.S. A.). Data were presented as means \pm SD and percentages for continuous and categorical variables, respectively. The categorial variables were compared among the patients applying One-way analysis of variance (ANOVA), Kruskal Wallis chi-square test, Fisher exact tests when appropriate, and continuous variables were compared among the patients applying Students' T test and ANOVA.

Multivariate linear regression model was applied to study the association between CLC and the risk of initial GI involvement and virus discharge in stool. A dotted boxplot analysis was used to compare the difference in CLC distribution between the patients of COVID-19 with or without initial GI involvement. The difference in CLC was compared between the patients of positive and negative PCR results in stool. The receiver operation curve (ROC) was used to assess the predicting power of CLC and CRP on the virus discharge in stool. The CLC cutoff was calculated as sensitivity- (1- specificity).

\section{Results}

\section{Baseline Characteristics}

Of the 76 enrolled COVID-19 patients, 24 patients (31.5\%) presented with initial GI involvement on admission, including merely vomiting (7 cases, 29.2\%), merely diarrhea (11 cases, 45.8\%), and combined GI symptoms (6 cases, 25\%). When compared with the COVID-19 patients without initial GI involvement (WT) on admission, those with initial GI involvement $(\mathrm{WH})$ showed significantly higher pulse rate $(p=$ 0.033), higher rate of fatigue and dyspnea, $p=0.009$ and $p=0.003$, respectively (Table 1 ).

The patients of WH displayed a different profile of circulating inflammatory cells and biomarkers, when compared to the WT group. Significantly lower CLC ( $p=0.004)$ was found in WH group, while no differences were found in the circulating neutrophile count and plasma $C$ reactive protein (CRP) level. There was also no significant difference in positive rate of qRT-PCR on stool detecting SARS-CoV-2 between WH and WT (Table 1).

\section{Association between CLC and Initial GI Involvement}

The precise association between CLC and initial GI involvement was analyzed using multiple logistic regression model (Table 2). When the CLC increased to the 2nd and 3rd tertiles, the risk of initial GI 
involvement in crude model decreased by nearly $74 \%(\mathrm{OR}=0.26,95 \% \mathrm{Cl}: 0.07,0.96, \mathrm{p}=0.041)$ and nearly $85 \%(\mathrm{OR}=0.15,95 \% \mathrm{Cl}: 0.04,0.52, \mathrm{p}=0.003)$, respectively. When adjusted for probable confounders, including gender, age, CRP, albumin globulin ratio, hypertension and dyspnea, this negative association remained (Table 2, Model I and Model II).

A dotted boxplot was used to compare the distribution of CLC and plasma CRP level between WT and WH subgroups of COVID-19 (Fig. 1). CLC was found significantly down-regulated in WH ( $p=0.004)$, while no significant difference was found in the plasma CRP level. Significant lower CLC was found in the patients, who showed a positive result of PCR on stool in the course of COVID-19 (Fig. 1).

\section{Predictive Power of CLC on Virus Discharge in Stool}

ROC was used to assess the predicting power of CLC and CRP on the virus discharge in stool (Fig. 2). The area under curve of CLC is 0.72 ( $95 \% \mathrm{Cl}: 0.59,0.85, \mathrm{p}=0.007$ ), while that of CRP is 0.59 ( $95 \% \mathrm{Cl}: 0.45$, $0.73, p=0.267)$. The cutoff value for CLC is at $0.95 \times 10^{\wedge} 9 / I$ (sensitivity $=68.75 \%$, specificity $=67.8 \%$ ).

\section{Discussion}

Ever since the outbreak of SARS-CoV-2 in Wuhan city, China, it has been identified as highly contagious and deadly. Up to the end of June, 2020, over 10 million people were proclaimed infected and 500 thousand confirmed death worldwide, and the number would probably continue increasing.

Though SARS-CoV-2 was identified as pulmonary pathogen, several studies have shown that the virus nucleus could be detected in stool in the disease process, indicating virus discharge via GI tract with the underline mechanism unknown $(3,7)$. What's worse, no reliable and convenient biomarker was available to predict the risk of initial GI involvement and virus discharge in stool of the COVID-19 patient.

\section{Correlation between CLC and Initial GI Involvement}

In this retrospective observational study, a negative correlation between CLC and initial GI involvement on admission (Table 2) was found, independent of age, gender, CRP, albumin globulin ratio, hypertension, dyspnea. The angiotentsin receptors were responsible for SARA-CoV-2 adhesion and invasion, which widely exists in the organs as pulmonary tract and GI tract $(8,9)$. Interestingly, not all of the COVID-19 patients displayed initial GI involvement. A negative correlation between CLC and the risk of initial GI involvement, independent of hypertension.

When compared to the patients of bottom tertile of CLC, the risk of initial GI involvement decreased dramatically in the 2nd and 3rd tertile, supporting the protective role of CLC against initial Gl involvement mentioned above. It's reported that the virus initially incubated in the pulmonary tract and GI tract, and then leaded to clinical symptoms if the cellular immune system was defeated, which was supported by our findings. That is to say, when the CLC declined in the course of COVID-19, more attention should be 
paid to virus discharge via GI tract. Our result was further supported by the findings elsewhere. Mei Jiang (10) et a/ found that T subset of lymphocyte count in peripheral blood is down regulated in the cases of severe COVID-19. Remes Troche (11) et a/ reported that SARS-CoV-19 has shown the probability to invade the mucosal of $\mathrm{Gl}$, leading to watering and virus discharge. And furthermore, we found that lower CLC is significantly associated with positive PCR results in stool (Fig. 2), indicating a positive role of CLC in preventing virus discharge via GI tract. More interestingly, no significant difference in the positive rate of qRT-PCR on stool was found between WT and WH in this research, indicating equal probability in virus discharge no matter GI symptoms were initially observed or not.

Higher CRP level has been reported as positively related to the severity of lung injury and COVID-19 severity by other researchers $(12,13)$. However, in our study, no significant correlation between plasma CRP level and the risk of initial GI involvement has been found in this study (Table 1). That indicates, for the first time, CLC is better than plasma CRP level in predicting initial GI involvement in COVID-19 patients, which should be taken into mind by the clinicians.

\section{Predictive Power of CLC in Virus Discharge in Stool}

No significant differences were found about the positive rate of qRT-PCR in stool between WH and WT groups, indicating the virus might discharge through GI tract no matter Gl initially involved or not.

Therefore, a biomarker is needed urgently to predict virus discharge in stool. The predictive power on virus discharge via GI tact was analyzed using ROC curve. Finally, a better performance in predicting virus discharge in stool has been found in CLC, when compared with that of CRP (Fig. 2). That indicates, in combination, CLC is a better biomarker in predicting initial Gl involvement and virus discharge in stool of COVID-19 patient.

The major limitation of this research is the relatively small volume of sample, and two-centers research, which might lead to possible enrollment bias. And the major strengthen is that the research is the first study on the risk factors on initial Gl involvement and virus discharge in stool.

\section{Conclusions}

In conclusion, lower CLC is negatively associated with virus discharge in stool in COVID-19 patients, and higher risk of initial GI involvement also.

\section{Declarations}

Acknowledgements:

The authors would like to pay acknowledgements to all of the clinicians, nurses, laboratory clinicians, and the public health staffs collaborating in combatting and preventing COVID-19 in this epidemic era.

Conflict of interest: 
The authors declared no conflict of interest.

Ethics Approval and Consent to Participate:

This study has been approved by the Ethics of Committees of the Sixth Hospital of Wenzhou, and the Second Affiliated Hospital of Wenzhou Medical University, and in accordance with the 1964 Helsinki Declaration and its later amendments or comparable ethical standards. Informed consent for participation in this retrospective study and publication was written and obtained.

Consent to participate:

Applicable.

Consent for Publication:

Applicable.

Availability of data and material:

Applicable when requested.

Authors' contributions:

Wei Chen and Zengpei Qiao designed the study.

Saiduo Liu and Chongyong Xu took part in diagnosis and sample collection. Zengpei Qiao and Chao Xing did the statistical analysis.

Wei Chen and Zengpei Qiao wrote the draft.

Kenneth I. Zheng performed critical review of the manuscript. All authors reviewed and approved the final version.

\section{References}

1. Zhang W, Du RH, Li B, Zheng XS, Yang XL, Hu B, et Molecular and serological investigation of 2019nCoV infected patients: implication of multiple shedding routes. Emerg Microbes Infect. 2020;9(1):386-9.

2. Di Wu TW, Qun Liu, et al. The SARS-CoV-2 outbreak_: what we know? Int J Infect Dis. 2020;94:44-8.

3. Holshue ML, DeBolt C, Lindquist S, Lofy KH, Wiesman J, Bruce H, et al. First Case of 2019 Novel Coronavirus in the United New England Journal of Medicine. 2020;382(10):929-36.

4. JingCheng Zhang SW, YaDong Xue. Fecal specimen diagnosis 2019 Novel Coronavirus Infected_ Pneumonia. J Med 2020. 
5. China Center for Disease Control and Diagnosis and Treatment of COVID-19 in China (seventh edition). In Chinese. Published March 5, 2020. Accessed March 13, 2020. .

6. Zhou P, Yang XL, Wang XG, Hu B, Zhang L, Zhang W, et al. A pneumonia outbreak associated with a new coronavirus of probable bat origin. Nature.

7. Wei X-S, Wang X, Niu Y-R, Ye L-L, Peng W-B, Wang Z-H, et al. Diarrhea Is Associated With Prolonged Symptoms and Viral Carriage in Corona Virus Disease 2019. Clinical Gastroenterology and 2020.

8. Oberoi M, Grover A. A systematic review and meta-analysis to evaluate the clinical outcomes in COVID-19 patients on angiotensin-converting enzyme inhibitors or angiotensin receptor blockers. European Heart Journal - Cardiovascular 2020.

9. Akoumianakis I, Filippatos T. The renin-angiotensin-aldosterone system as a link between obesity and coronavirus disease 2019 Obes Rev. 2020.

10. Mei Jiang YG, Qing Luo, et al. . T cell subset counts in peripheral blood can be used as discriminatory biomarkers for diagnosis and severity prediction of COVID-19. J Infect Dis. 2020 May

11. Remes T, María J, Ramos de la M, Antonio, Manríquez R, Marisol, et al. Initial Gastrointestinal Manifestations in Patients with SARS-CoV-2 in 112 patients from Veracruz (Southeastern Mexico). 2020.

12. Wei Chen KIZ, Saiduo Liu, Zhihan Yan, Chongyong Xu, Zengpei Qiao. Plasma CRP level is positively associated with the severity of COVID-19. Ann Clin Microbiol Antimicrob. 2020;19 (1)(18).

13. Xiaomin Luo WZ, Xiaojie Yan, et al. Prognostic value of C-reactive protein in patients with COVID-19. Clin Infect Dis.

\section{Tables}

Table 1. Baseline characteristics of included patients, stratified by initial gastrointestinal involvement. 


\begin{tabular}{|c|c|c|c|}
\hline & WT $(n=52)$ & WH $(n=24)$ & $p$-value \\
\hline \multicolumn{4}{|l|}{ Demographics } \\
\hline Age (years) & $44.3 \pm 12.6$ & $45.1 \pm 13.4$ & 0.807 \\
\hline Male (\%) & $24(46.2)$ & $10(41.7)$ & 0.715 \\
\hline Higest Temperature (degree centigrade) & $38.0 \pm 0.7$ & $38.3 \pm 0.5$ & 0.095 \\
\hline Pulse per minute & $90 \pm 10$ & $96 \pm 12$ & 0.033 \\
\hline Hypertension (\%) & $11(21.15)$ & $7(29.17)$ & 0.445 \\
\hline Fatigue (\%) & $20(38.46)$ & $17(70.83)$ & 0.009 \\
\hline \multicolumn{4}{|l|}{ Respiratory Manifestation } \\
\hline Breath (times per minute) (\%) & & & 0.066 \\
\hline 16 & $1(1.92)$ & $0(0.00)$ & \\
\hline 17 & $1(1.92)$ & $0(0.00)$ & \\
\hline 18 & $5(9.62)$ & $0(0.00)$ & \\
\hline 19 & $1(1.92)$ & $4(16.67)$ & \\
\hline 20 & $38(73.08)$ & $15(62.5)$ & \\
\hline 21 & $2(3.85)$ & $1(4.17)$ & \\
\hline 22 & $4(7.69)$ & $2(8.33)$ & \\
\hline 23 & $0(0.00)$ & $2(8.33)$ & \\
\hline Dyspnea (\%) & $5(9.62)$ & $9(37.5)$ & 0.004 \\
\hline Cough (\%) & $32(61.54)$ & $18(75)$ & 0.250 \\
\hline \multicolumn{4}{|l|}{ Laboratory Characteristics } \\
\hline White Blood Cell count $\left(10^{\wedge} 9 / \mathrm{I}\right)$ & $4.57 \pm 1.52$ & $4.21 \pm 1.41$ & 0.335 \\
\hline Neutrophile Count $\left(10^{\wedge} 9 / \mathrm{I}\right)$ & $2.87 \pm 1.20$ & $3.06 \pm 1.27$ & 0.534 \\
\hline Lymphocyte count $\left(10^{\wedge} 9 / \mathrm{I}\right)$ & $1.24 \pm 0.53$ & $0.88 \pm 0.38$ & 0.004 \\
\hline Hemoglobulin (g/l) & $137.79 \pm 11.40$ & $133.63 \pm 15.74$ & 0.195 \\
\hline Platelet count $\left(10^{\wedge} 9 / \mathrm{I}\right)$ & $180.81 \pm 64.66$ & $164.13 \pm 60.72$ & 0.116 \\
\hline C Reactive Protein (mg/l) & $16.96 \pm 21.30$ & $27.99 \pm 25.41$ & 0.052 \\
\hline Total Protein (g/l) & $71.36 \pm 5.85$ & $70.12 \pm 5.56$ & 0.385 \\
\hline Globulin $(\mathrm{g} / \mathrm{l})$ & $29.86 \pm 5.24$ & $29.46 \pm 4.24$ & 0.745 \\
\hline
\end{tabular}




\begin{tabular}{|llll|}
\hline Albumin Globulin Ratio & $1.43 \pm 0.27$ & $1.41 \pm 0.25$ & 0.719 \\
\hline Lactic dehydrogenase (U/L) & $216.37 \pm 70.32$ & $248.13 \pm 90.66$ & 0.100 \\
\hline Creatinine $(\mathrm{mmol} / \mathrm{I})$ & $69.50 \pm 15.02$ & $73.32 \pm 18.55$ & 0.330 \\
\hline Alanine Aminotranferase (U/L) & $28.73 \pm 26.19$ & $33.00 \pm 24.25$ & 0.501 \\
\hline Aspartate transaminase (U/L) & $31.65 \pm 18.77$ & $34.67 \pm 27.62$ & 0.579 \\
\hline Total Bilirubin (mmol/l) & $15.32 \pm 8.36$ & $13.80 \pm 8.65$ & 0.470 \\
\hline qRT-PCR on stool (\%) & $10(18.18)$ & $7(29.16)$ & 0.334 \\
\hline
\end{tabular}

Note: qRT-PCR: quantitative real-time Polymerase chain reaction; WT: Patients of COVID-19 presented without gastrointestinal tract affected on admission; WH: Patients of COVID-19 presented with gastrointestinal tract affected on admission.

Data are presented as means $\pm \mathrm{SD}$, or $\mathrm{n}(\%)$.

Table 2. Relationship between circulating lymphocyte count and initial gastrointestinal involvement.

\begin{tabular}{|clcccccc|}
\hline \multicolumn{5}{|l}{ Initial gastrointestinal involvement } & & & \\
Lymphocyte & Crude Model & & Adjusted Model I & \multicolumn{2}{l|}{ Adjusted Model II } & \\
\hline tertile & OR $(95 \% \mathrm{Cl})$ & p value & OR $(95 \% \mathrm{Cl})$ & p value & OR $(95 \% \mathrm{Cl})$ & p value \\
\hline 1 & 1 & & 1 & & 1 & \\
\hline 2 & $0.26(0.07,0.95)$ & 0.041 & $0.25(0.07,0.92)$ & 0.038 & $0.25(0.07,0.98)$ & 0.047 \\
\hline 3 & $0.15(0.04,0.52)$ & 0.003 & $0.14(0.04,0.52)$ & 0.003 & $0.17(0.05,0.63)$ & \\
& 0.008 & & & & & & \\
\hline
\end{tabular}

Crude Model adjusted for None.

Adjusted Model I adjusted for age, gender.

Adjusted Model II adjusted for age, gender, CRP, albumin globulin ratio, hypertension, dyspnea.

Lymphocyte Tertile $1<=0.88 * 10^{\wedge} 9 /$;

$0.88 * 10^{\wedge} 9 / \mathrm{l}<$ Lymphocyte Tertile $2<=1.2 * 10^{\wedge} 9 / \mathrm{l} ;$

Lymphocyte Tertile $3>1.2$ * 10^9/I.

\section{Figures}


$p=0.012$

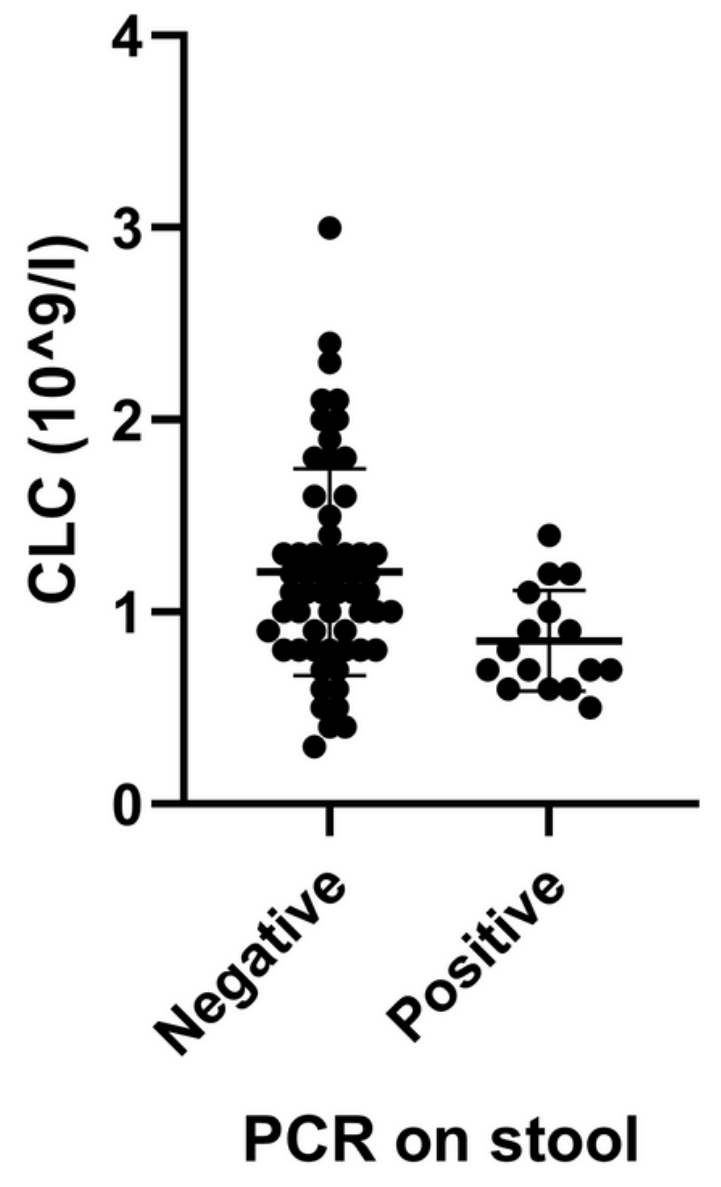

$p=0.004$

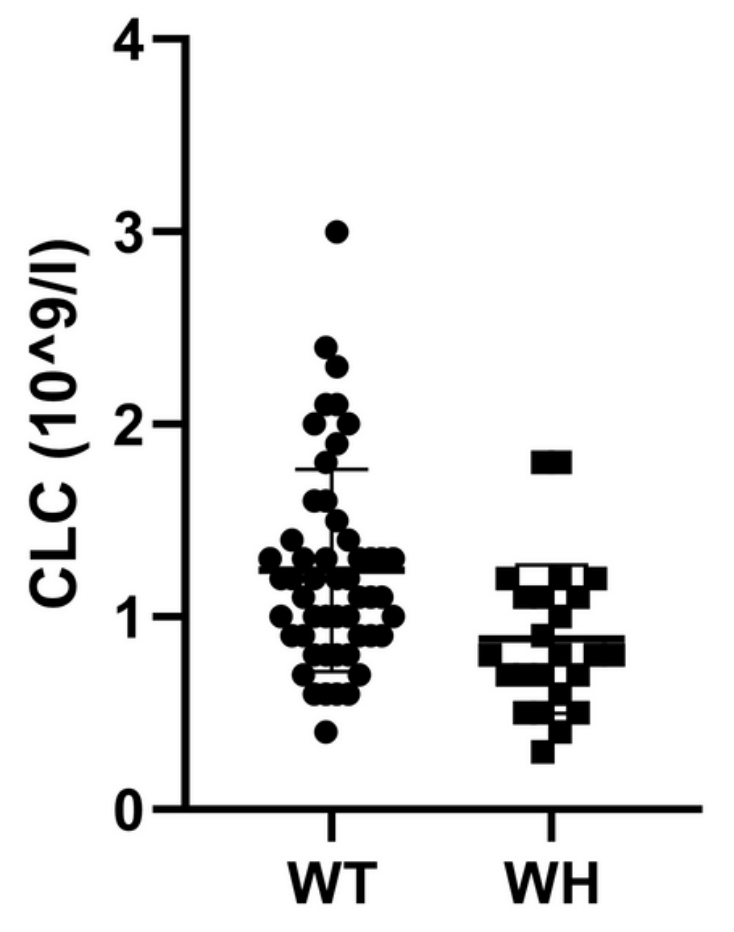

Initial GI involvement

Figure 1

Dotted boxplot analysis of CLC distributions. 
ROC curves on virus discharge in stool

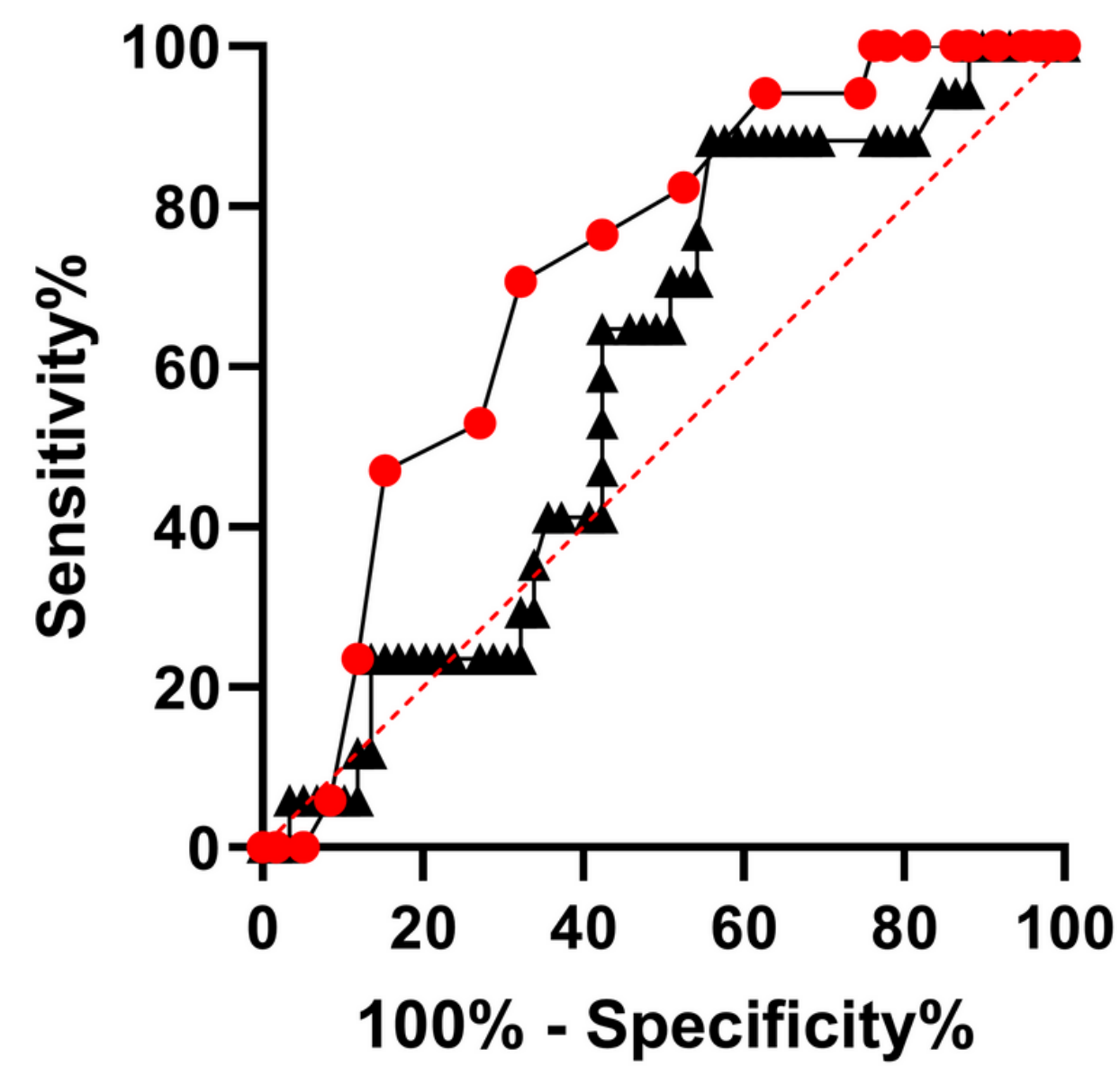

Figure 2

ROC curves of CLC and CRP. 